\title{
Fabrication of Aluminum/Single-Walled Carbon Nanotube Oxidation Films through CNT-Added Surface Treatment
}

\author{
Sangjae Kim¹, Emi Matsuyama1, Hideharu Tajima1, Rikuma Miyake², Takahiro Maruyama ${ }^{3,4}$ \\ ${ }^{1}$ Research \& Development Center, Yamaichi Special Steel, Nagoya, Aichi, Japan \\ ${ }^{2}$ Department of Applied Physics, Nagoya Institute of Technology, Nagoya, Aichi, Japan \\ ${ }^{3}$ Department of Applied Chemistry, Meijo University, Nagoya, Aichi, Japan \\ ${ }^{4}$ Nanomaterial Research Center, Meijo University, Nagoya, Aichi, Japan \\ Email: sangjae.kim@yamaichi-hagane.co.jp
}

How to cite this paper: Kim, S., Matsuyama, E., Tajima, H., Miyake, R. and Maruyama, T. (2022) Fabrication of Aluminum/Single-Walled Carbon Nanotube Oxidation Films through CNT-Added Surface Treatment. Journal of Surface Engineered Materials and Advanced Technology, 12, 1-13.

https://doi.org/10.4236/jsemat.2022.121001

Received: December 6, 2021

Accepted: January 8, 2022

Published: January 11, 2022

Copyright $\odot 2022$ by author(s) and Scientific Research Publishing Inc.

This work is licensed under the Creative

Commons Attribution International

License (CC BY 4.0).

http://creativecommons.org/licenses/by/4.0/

\begin{abstract}
CNT-added surface treatment (CAST) is a newly developed technology that incorporates single-walled carbon nanotubes (SWCNTs) into a metal surface through alternate current electrolysis using a dispersion of SWCNTs in an alkaline aqueous solution. We apply this method to Al-plates and characterize their surface morphology and components through scanning electron microscopy (SEM), transmission electron microscopy (TEM), X-ray photoelectron spectroscopy (XPS), and Raman spectroscopy. After CAST processing, protrusion structures of Al-oxide containing SWCNTs are formed on the surface of the Al-plate, and the surface morphology differs significantly from that of the surface of Al films treated through conventional anodic oxidation. The height and spacing of the protrusion structures formed on the surface of the CAST-treated Al-plates are $100-200 \mathrm{~nm}$ and $50-100 \mathrm{~nm}$, respectively. In addition, we investigate the formation mechanism of the protrusion structure by applying a DC voltage between the working electrode (Al-plate) and a counter electrode immersed in a dispersion of SWCNTs in an alkaline aqueous solution. Comparing the Al-plate surface after treatment under both current directions, we propose a model for the formation process of protrusion structures containing SWCNTs based on catalyst surface etching.
\end{abstract}

\section{Keywords}

SWCNT, Anodic Oxidation, Anodic Aluminum Oxide

\section{Introduction}

Carbon nanotubes (CNTs), with concentric wall structures composed of rolled 
graphene sheets, exhibit unique mechanical, thermal, optical, electrical, and chemical properties rendering them ideal candidates for various applications such as composite materials, sensors, field emission displays, and integrated circuits [1]-[12]. In particular, CNTs have a super-high thermal conductivity of the order of $1000 \mathrm{~W} \cdot \mathrm{m}^{-1} \cdot \mathrm{K}^{-1}$ at $298 \mathrm{~K}$ [13]. The fabrication of polymer/CNT composite [14] [15] [16] has been extensively researched because it shows better thermal transport than the host material. Moreover, as the addition of CNTs to a host material enhances its mechanical strength, CNTs have been added to metals such as $\mathrm{Cu}$ [17] [18] [19] [20] and $\mathrm{Al}$ [21]-[28].

Anodic oxidation is a common process for forming an oxide film on a metallic substrate through an electrochemical method. It can increase the corrosion resistance and wear resistance of the metal film and provide better adhesion for paint primers and glues than bare metal. In addition, Al-oxides formed through anodic oxidation on $\mathrm{Al}$ film have porous structures on the surface, which is beneficial for dye absorption or the addition of interference effects. However, studies on anodic oxidation for the dispersion of CNTs are limited.

In this study, we investigate the fabrication of Al-oxide layers containing single-walled carbon nanotubes (SWCNTs) on the surface of Al films through anodic oxidation using alkaline aqueous solutions. We refer to this method as CNT-added surface treatment (CAST). An SWCNT can be conceptually viewed as a graphene sheet rolled into a nanoscale tube [29] [30]. We demonstrate that protrusion structures of Al-oxide containing SWCNTs can be formed on Al-plates through CAST processing. In addition, we discuss the formation mechanism of the protrusion structures containing SWCNTs based on catalyst surface etching.

\section{Experimental}

\subsection{Apparatus for CAST Processing}

An Al-plate was CAST processed in $\mathrm{NaOH}$ solution containing SWCNTs, using an anodic oxidation measurement system (TEXIO Co., PSW-720M160). The experimental setup for CAST processing is shown in Figure 1. An Al-plate (>99.5\% purity, LSI Co., Ltd.) $\left(6 \times 6 \mathrm{~cm}^{2}\right)$, washed with ethanol for $5 \mathrm{~min}$, was employed as the substrate for CAST processing. An SUS304 plate (Yamamoto-Ms Co., Ltd.) $\left(7 \times 10 \mathrm{~cm}^{2}\right)$, which was also washed with ethanol for $5 \mathrm{~min}$, was employed as the counter electrode. For CAST processing, an aqueous dispersion of SWCNTs (approximately $6 \times 10^{-5} \mathrm{wt} \%$ ) purchased from KORBON Co. Ltd. (Gangwon-do, South Korea, Product Name: WAB1300ST-6P30) was used. It was diluted with $0.04 \mathrm{M} \mathrm{NaOH}$ solution, prepared with $\mathrm{NaOH}$ (97\% Assay, Yoneyama Yakuhin Kogyo, Ltd.), and used as the electrolyte.

As in normal anodic oxidation, the working electrode (electrode of the sample subjected to CAST treatment) and the counter electrodes were immersed in the solution. The same voltage was applied to the two electrodes used as the counter electrodes during CAST processing because the same treatment was applied to both sides of the working electrode. 


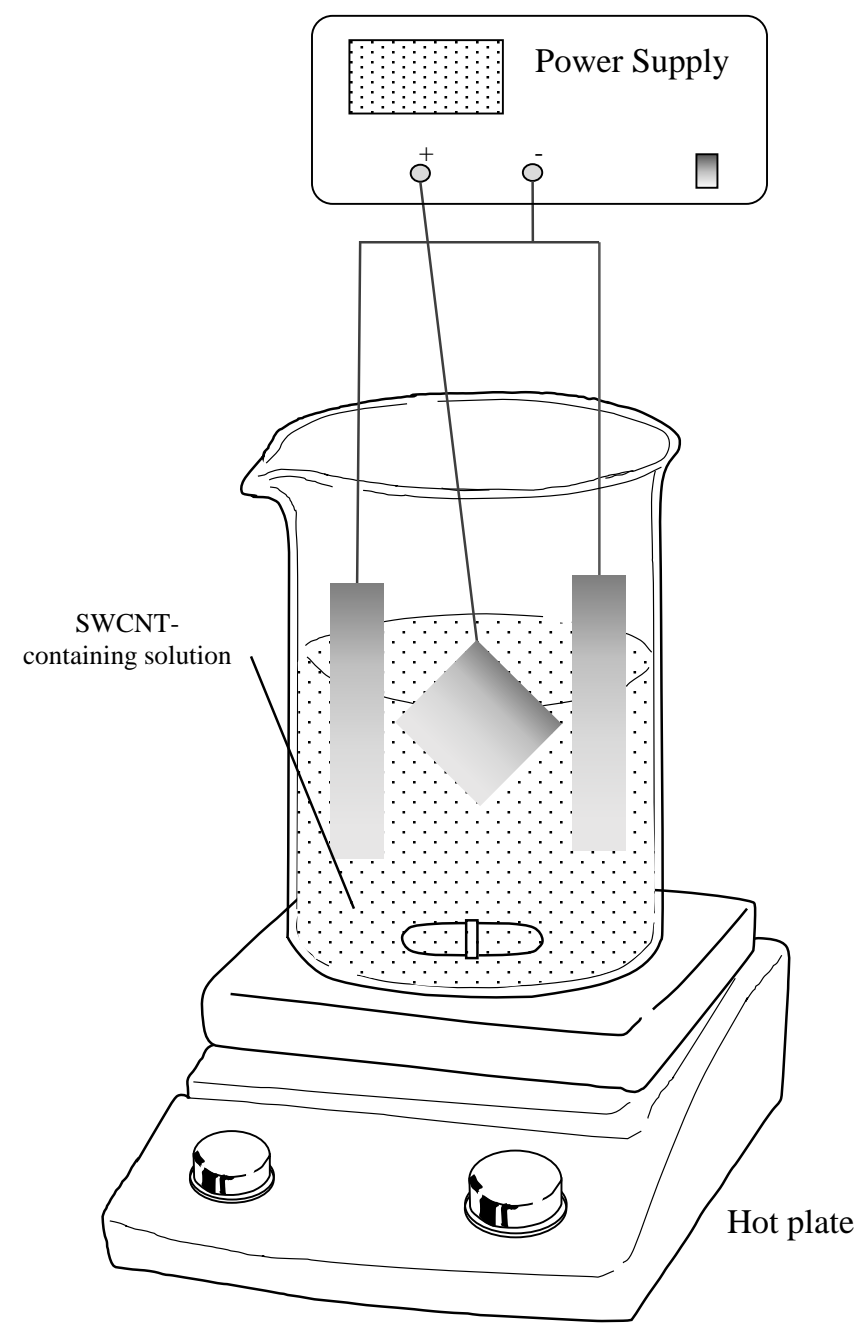

Figure 1. Schematics of apparatus for CAST processing.

The current waveform applied during CAST processing is displayed in Figure 2. Unlike conventional anodic oxidation, the current direction was changed alternatively with time. When the current flowed along the positive direction, the working electrode was the anode; however, when the current flowed in the opposite direction, it became the cathode.

\subsection{Characterization}

After CAST processing, the surface morphology of the Al-plate was characterized through field-emission scanning electron microscopy (FE-SEM, Hitachi Co., Ltd., SU8010) at an accelerating voltage of $5 \mathrm{kV}$. In addition, AFM analysis (Bruker AXS Dimension 3100) was performed to investigate the surface shapes of the specimens. The frequency range was set to $200-400 \mathrm{kHz}$. Raman spectra from $300-2000 \mathrm{~cm}^{-1}$ were recorded at $298 \mathrm{~K}$ using a Raman spectroscope (Nanophoton, Japan), at an excitation wavelength of $532 \mathrm{~nm}$. Furthermore, to analyze the surface composition [31], X-ray photoelectron spectroscopy (XPS) was performed on the Al-plate after CAST processing using an XPS system (JEOL 


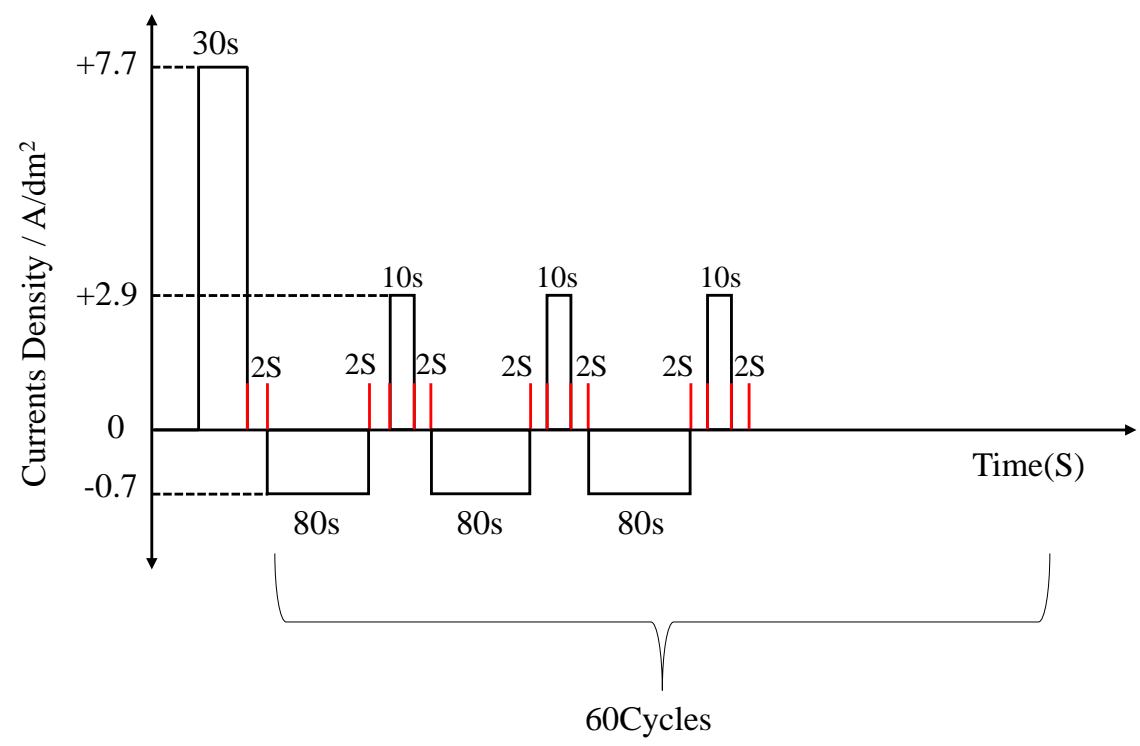

Figure 2. Current waveform applied during CAST processing.

JPS-9200, Japan); an Mg K $\alpha$ source (1253.6 eV) was used for XPS analysis. Scanning transmission electron microscopy (STEM) was performed at an accelerating voltage of $200 \mathrm{kV}$ for observing the fine structures on the sample cross-section (JEOL ARM-200F, Japan). To investigate the composition distribution, energy-dispersive X-ray spectroscopy (EDX) was performed using an EDX spectrometer (JEOL JED-2300T, Japan), mounted on the TEM.

\section{Results and Discussion}

\subsection{Electron Microscopy and Atomic Force Microscopy Observations}

Figure 3(a) depicts the Al-plate surface after CAST processing ("CAST-treated Al-plate"). For comparison, the surface of the Al-plate after anodic oxidation with the same current waveform in $\mathrm{NaOH}$ solution without SWCNTs is shown in Figure 3(b). The surface of the Al-plate subjected to CAST processing is mostly protrusive. In addition, fibrous substances bridged between the protrusions can be observed. On the other hand, in the Al-plate subjected to anodic oxidization (Figure $3(\mathrm{~b})$ ), a porous structure is formed on the surface, and the diameters of most of the holes are approximately $10 \mathrm{~nm}$. It is to be noted that no protrusions are observed on the surface of the anodic oxidation Al-plate. This result indicates that CAST processing is effective in forming a protrusion microstructure on the Al-plate surface.

Furthermore, the surface of the Al-plate subjected to anodic oxidation and the CAST-treated Al film were analyzed through AFM. The AFM 3D images of the Al-plates after CAST processing and anodic oxidation are depicted in Figure 4(a) and Figure 4(b), respectively. Consistent with the SEM observation, a protrusion structure is observed on the CAST-treated Al-plate, where the height of the protrusion and the spacing are approximately $100-200 \mathrm{~nm}$ and $50-100 \mathrm{~nm}$, 


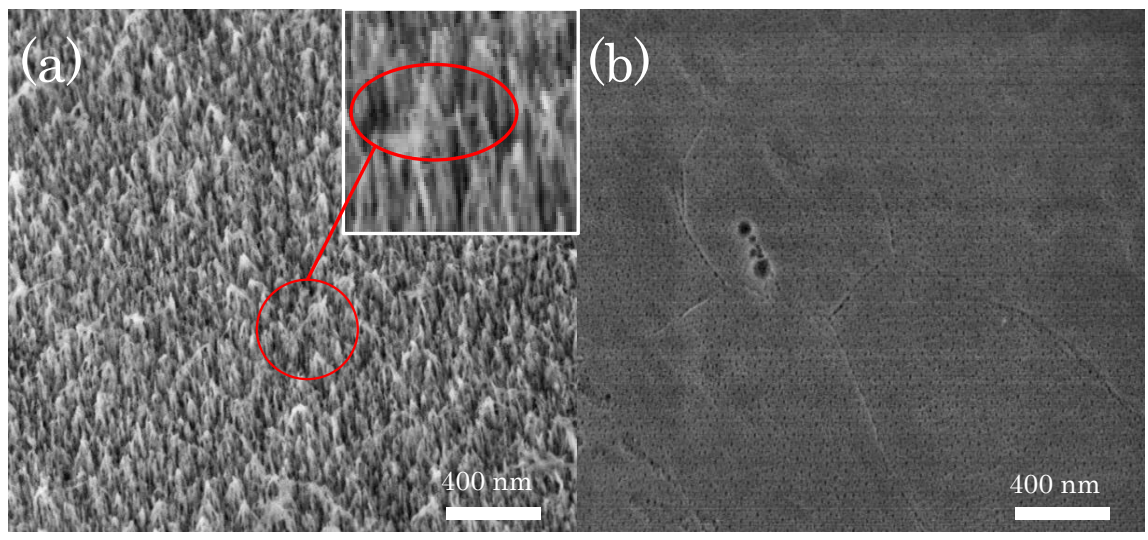

Figure 3. SEM images of the (a) CAST-treated Al-plate, and (b) Anodic Oxidation Al-plate.
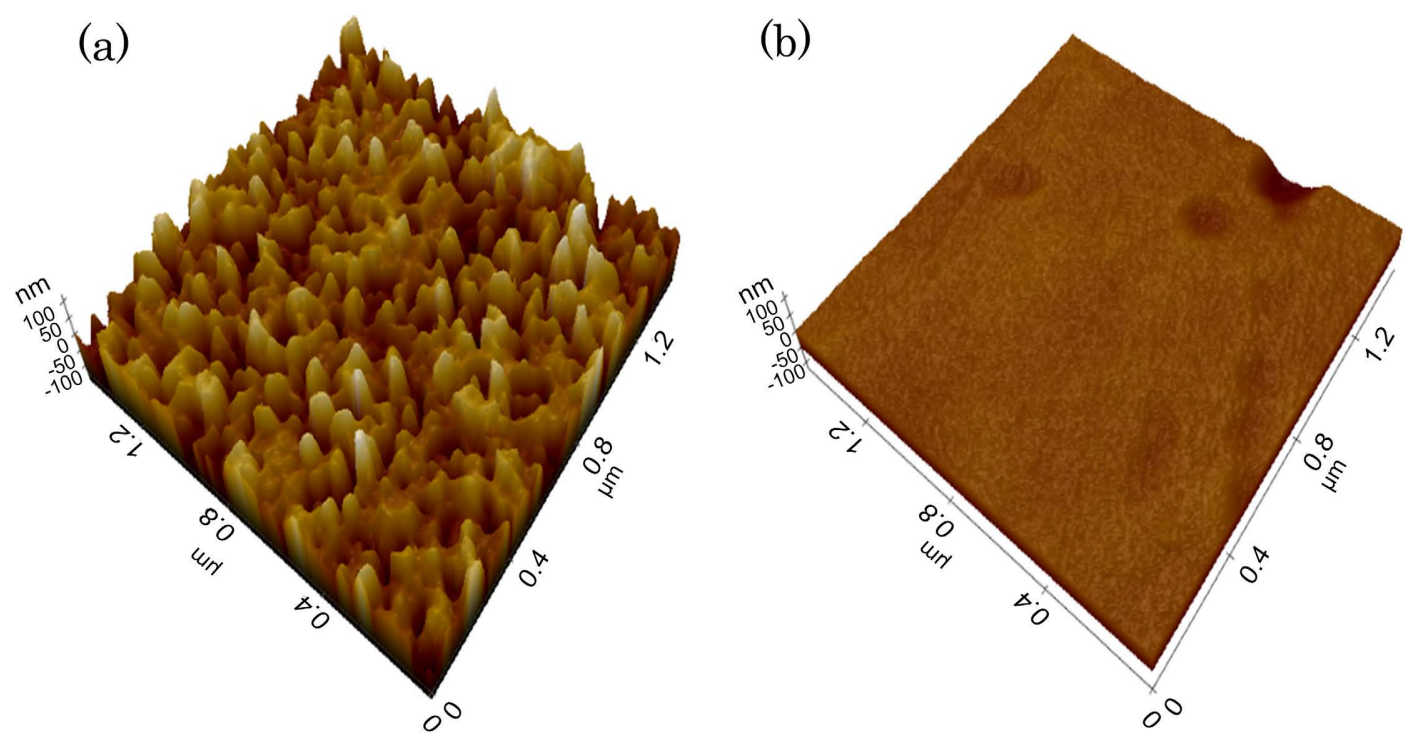

Figure 4. AFM images of the (a) CAST-treated Al-plate, and (b) Anodic oxidation Al-plate (scan range $1.5 \times$ $1.5 \mu \mathrm{m})$.

respectively. In contrast, there are no protrusions on the surface of the Al-plate subjected to anodic oxidation, and the observed areas are relatively flat. Contrary to the SEM observation, holes are not observed in the AFM image, which may be due to the larger diameter of the AFM probe than those of the holes.

\subsection{Raman Spectroscopy Analysis}

Figure 5(a) and Figure 5(b) display the low-frequency and high-frequency regions, respectively, of the Raman spectra of the CAST-treated Al-plate. In the low-frequency region, $\mathrm{Al}_{2} \mathrm{O}_{3}$ derived peaks are observed at approximately 359, 453 , and $612 \mathrm{~cm}^{-1}$, whereas G-band and D-band peaks appear at $1326 \mathrm{~cm}^{-1}$ and $1593 \mathrm{~cm}^{-1}$, respectively, in the high-frequency region. As the G-band, at approximately $1580 \mathrm{~cm}^{-1}$, is related to the $\mathrm{sp}^{2}$ hybrid graphite structure, indicating $\mathrm{C}=\mathrm{C}$ stretching vibrations [32], this establishes that Al-oxide films containing SWCNTs are formed on the CAST-treated Al surface. 

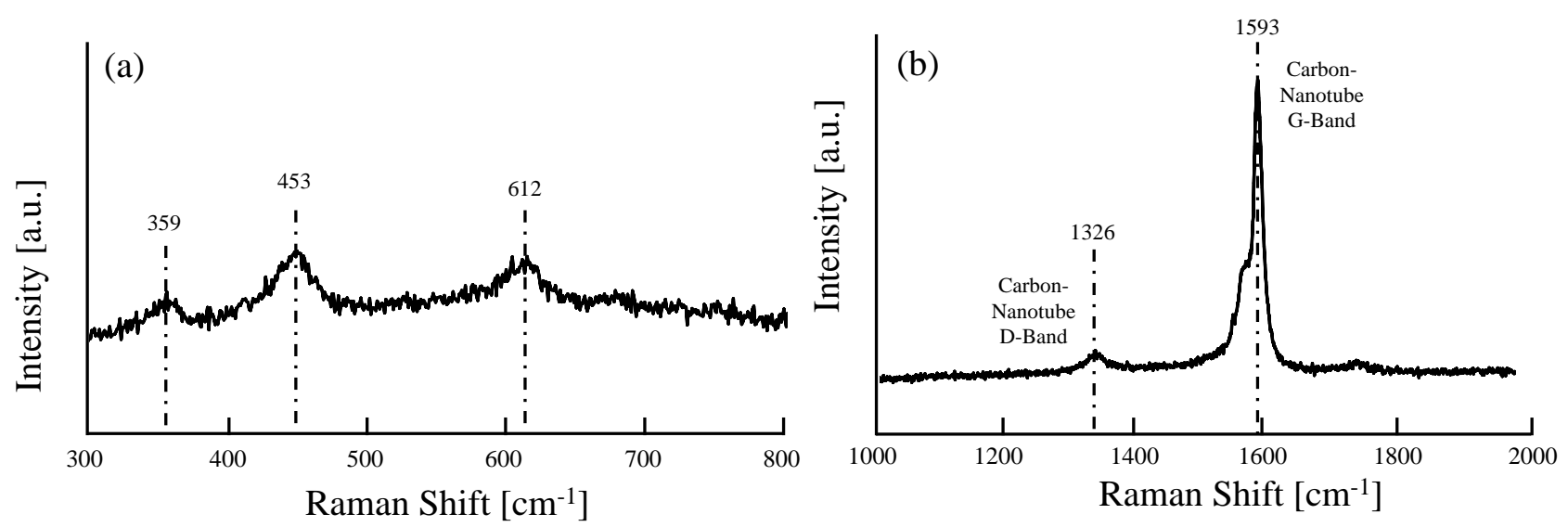

Figure 5. Raman spectra of the CAST-treated Al-plate: (a) low-frequency and (b) high-frequency regions. The excitation wavelength is $532 \mathrm{~nm}$.

\subsection{X-Ray Photoelectron Spectroscopy (XPS) Analysis}

XPS analysis is an analytical technique that can provide useful information on the functionalities of oxidized nanotubes. The XPS survey spectrum of the CAST-treated Al-plate is shown in Figure 6. To investigate the subsurface composition, XPS measurements were performed on the CAST-treated Al-plate after Ar-ion etching, corresponding to an area at a depth of $5 \mathrm{~nm}$. The resultant XPS spectrum in Figure 6 shows that in addition to $\mathrm{Al}$ and $\mathrm{O}$ derived peaks, a peak derived from $\mathrm{sp}^{2} \mathrm{C}-\mathrm{C}$ exists at $284.3 \mathrm{eV}$ [32] [33].

This suggests that SWCNTs are incorporated into the Al-oxide layer through CAST processing. Table 1 lists the chemical composition analysis results of the subsurface of the CAST-treated Al-plate, estimated from the XPS spectra in Figure 6. As $\mathrm{Al}: \mathrm{O}=2: 3$ in $\mathrm{Al}_{2} \mathrm{O}_{3}$, the result indicates that approximately $62 \%$ of the surface $\mathrm{Al}$ is oxidized.

\subsection{Scanning Transmission Electron Microscopy (STEM) Analysis}

The incorporation of SWCNTs into the CAST-treated Al-plate was investigated through STEM (High-angle annular dark field, HAADF) and EDX on the cross-section of the CAST-treated Al-plate. The obtained results for the cross-section near the surface are shown in Figure 7. In the STEM images of the CAST-treated Al surface in Figure 7(a), the protective layer deposited for STEM observation is observed on the top layer, and a protruding surface structure is observed on the surface of the CAST-treated Al-plate. Figure 7(b) shows the EDX mapping of carbon, oxygen, and $\mathrm{Al}$ on the surface area of the CAST-treated Al-plate. Based on the EDX results, the Al-oxide surface is divided into two regions: Diff-1 and Diff-2 (Figure 7(a)). Diff-1 shows porous structures with a thickness of $106.8 \mathrm{~nm}$, whereas Diff- 2 is an oxide film formed on the $\mathrm{Al}$ surface with a thickness of $31.7 \mathrm{~nm}$. It should be noted that carbon is also distributed around the porous area, and regardless of its shape, it is present. This confirms that SWCNTs are embedded, and a porous structure is formed. 


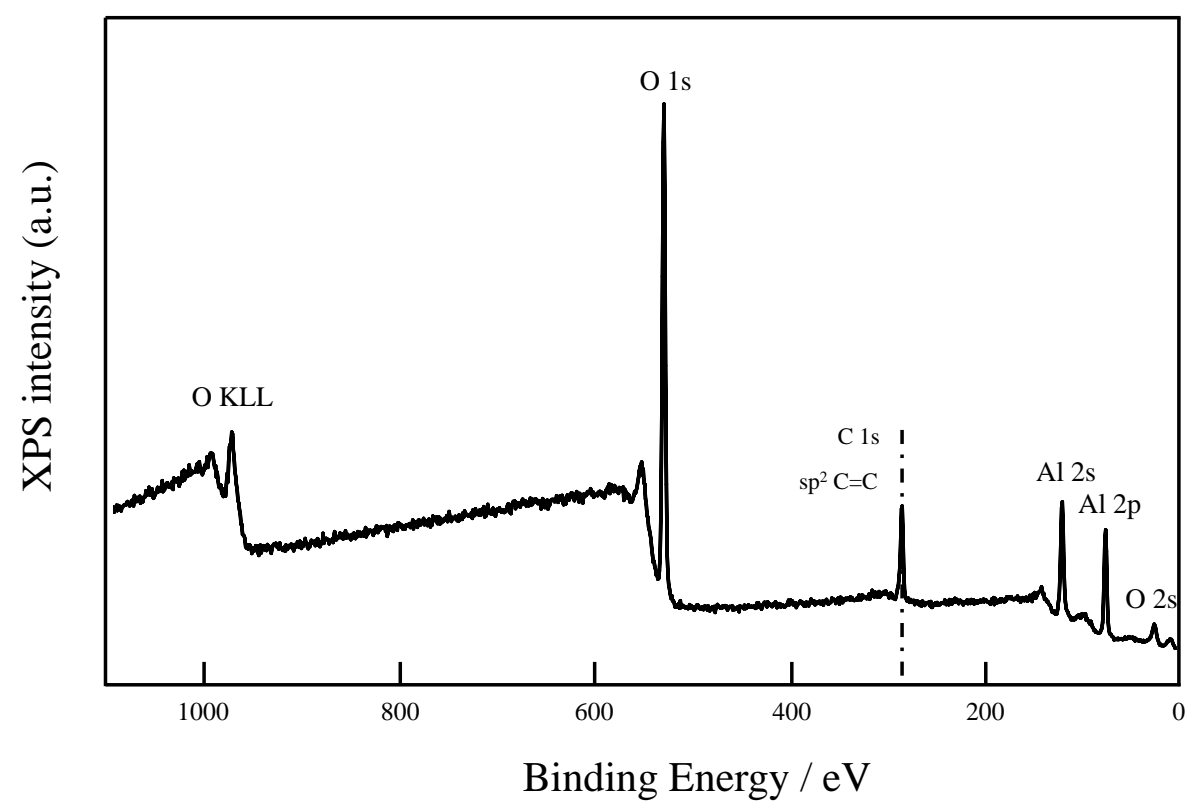

Figure 6. XPS survey spectrum of the CAST-treated Al-plate. The spectrum is measured in an area at a depth of $5 \mathrm{~nm}$.

(a)
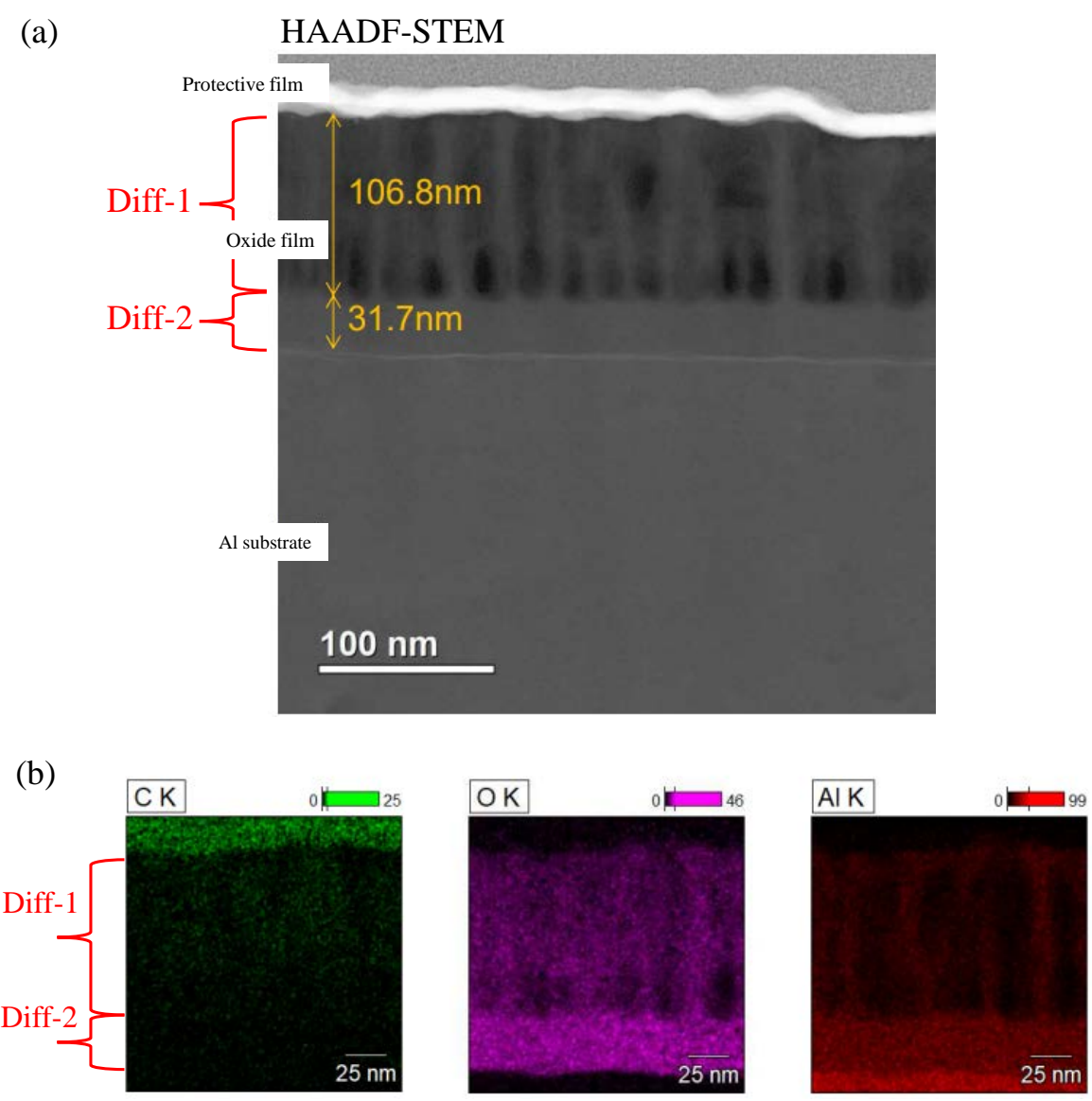

Figure 7. (a) STEM cross-section images (HAADF image) of the CAST-treated Al-plate. (b) EDX Mapping of carbon, oxygen, and $\mathrm{Al}$ on the surface area of the CAST-treated Al-plate. 
Table 1. Chemical composition of the CAST-treated Al-plate estimated from the XPS spectrum. The detection area is $5 \mathrm{~nm}$ from the surface.

\begin{tabular}{ccc}
\hline $\mathrm{Al}(\mathrm{at} \%)$ & $\mathrm{O}($ at $\%)$ & $\mathrm{C}($ at $\%)$ \\
\hline $42.8 \%$ & $45.7 \%$ & $11.5 \%$ \\
\hline
\end{tabular}

\subsection{CAST Mechanism}

The formation of a protrusion structure of Al-oxide containing SWCNTs through CAST processing is an interesting phenomenon. We consider this to be caused by catalyst surface etching [34], in which a catalyst on the surface of the material induces etching through the reduction of the material (mainly a metal) with an oxidizing agent in a solution. Figure 8 outlines the catalyst surface etching mechanism.

When the catalyst is attracted to the surface of the workpiece and contacts with it, the transfer of electrons from the workpiece to the oxidant in the solution is facilitated by the catalyst. The part in contact with the catalyst is oxidized and dissolves in the solution. By continuing this process, the surface of the workpiece is drilled by the catalyst, and as a result, a porous structure is formed on the surface of the workpiece.

With this method, selective surface treatment is possible because only the part in contact with the catalyst is superimposed. Semiconductors (such as $\mathrm{Si}$ or $\mathrm{Ge}$ ) are generally used as the workpiece material, and precious metals (such as Pt or $\mathrm{Au}$ ) are often used as catalysts. In addition, catalyst surface etching using graphene as a catalyst has been reported [34]. As CNTs are nanocarbon materials, such as graphene, there is a possibility that they function as a catalyst in the catalyst surface etching process. Furthermore, the workpiece is not limited to semiconductors, and metals can be used if oxidation occurs. Therefore, the protrusion structure may be formed through the same mechanism even in $\mathrm{Al}$, where a porous structure is generally formed through anodic oxidation.

In our experiment, an alternating voltage was applied between the working and counter electrodes, and the electric current flowed between them alternately. When the working electrode was the anode (positive bias), the current flow direction was the same as that of anodic oxidation treatment; therefore, an oxide layer was deposited on the working electrode.

On the other hand, it is not possible to determine the process that occurs when the working electrode is the cathode (negative bias). Based on catalyst surface etching, we suppose that CNTs function as a catalyst that etches and forms protrusion structures on the Al-film surface.

To verify this hypothesis, we performed electrolysis for $\mathrm{Al}$ films with direct current flow using the same apparatus shown in Figure 1, (CNT concentration: $0.2 \mathrm{wt} \%$, electrode: SUS, the distance between the electrodes: $2 \mathrm{~cm}$ ), where positive and negative bias voltages, respectively, were applied to the working electrode with respect to the counter electrode. Here, when the working electrode is positive, the current direction is the same as that of anodic oxidation, and when 
the working electrode is negative, the current direction is opposite to that of anodic oxidation. SEM images of the $\mathrm{Al}$ film surface after electrolysis with direct current are shown in Figure 9. When the working electrode is positive, a porous structure sized approximately $5-10 \mathrm{~nm}$ is formed, which is often observed in the anodic oxidation of Al films; however, SWCNTs are not found (Figure 9(a)). On the other hand, when the working electrode is negative, several SWCNTs are observed on the working electrode. This indicates that the SWCNTs are positively charged when they are dispersed in $\mathrm{NaOH}$ solution and are, therefore, repelled by when the working electrode is the anode.

Based on the results depicted in Figure 9, we propose a mechanism for the formation of the protrusion structure on Al films through CAST processing (shown in Figure 10). SWCNTs dispersed by a strong alkaline aqueous solution have a positive electric charge; therefore, when the working electrode (Al-plate) is the anode, the SWCNTs are repelled by it; the SWCNTs are attracted to the counter electrode and incorporated into the solution. In this case, anodic oxidation occurs on the surface of the Al-plate, and the oxide film grows. When the current flows in the opposite direction and the Al-plate becomes the cathode, the SWCNTs are attracted to the Al-plate. The surface of the Al-plate in contact with the CNTs is etched through catalyst surface etching. Simultaneously, the $\mathrm{Al}^{3+}$ ions dissolved in the solution accompany the SWCNTs and precipitate on the surface of the Al-plate. During alternating current flow, this process is repeated, and the SWCNTs contact at different angles several times, forming a protruding structure [35] [36] [37].

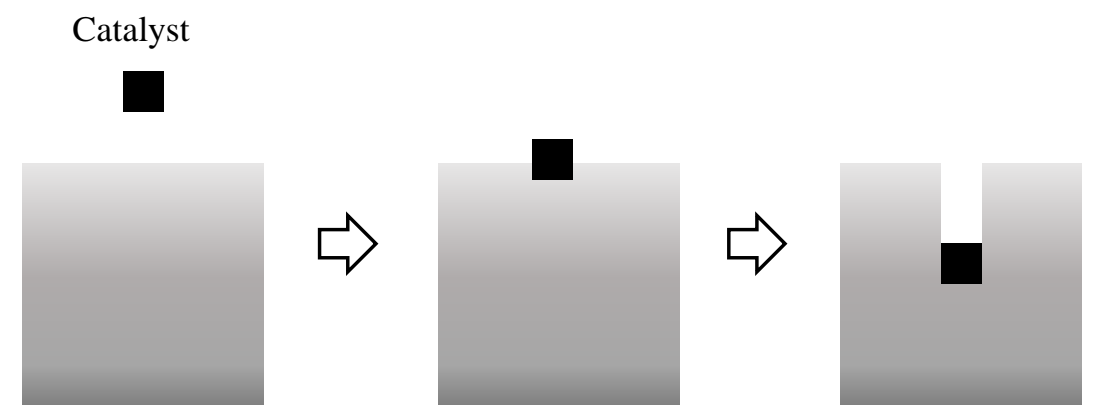

Figure 8. Catalyst surface etching mechanism.

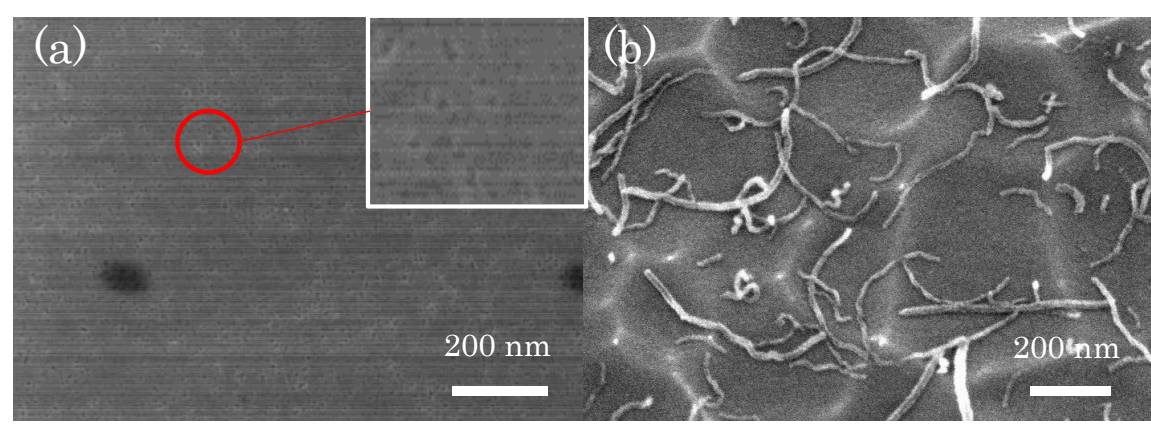

Figure 9. SEM image of the Al-plate surface during electrolysis: (a) same direction as anodic oxidation, and (b) direction opposite to anodic oxidation. 


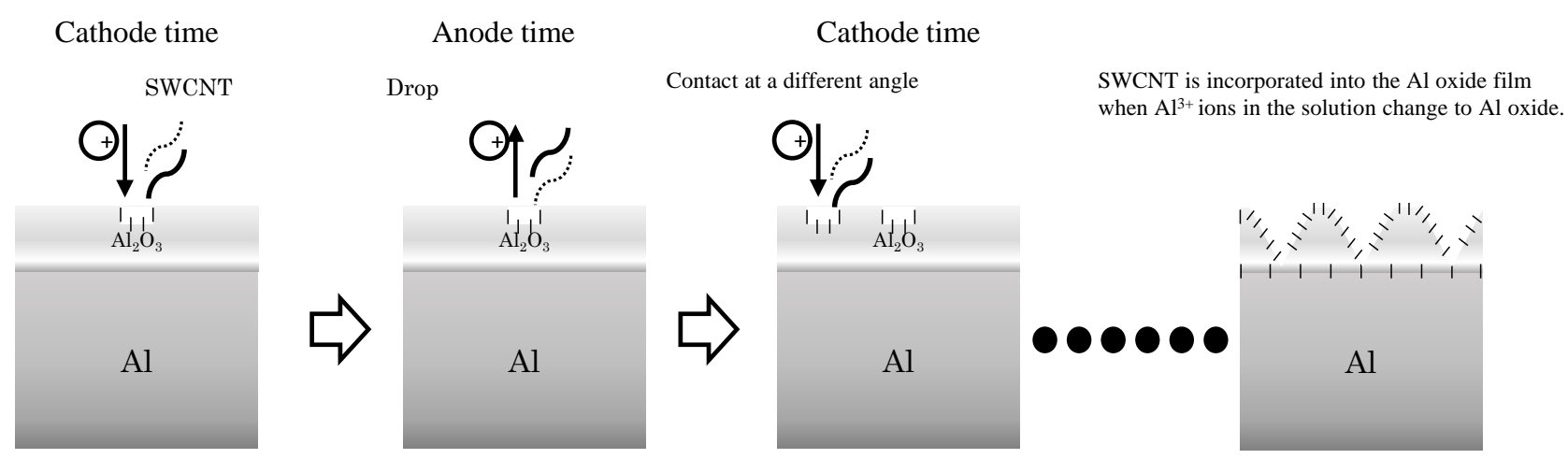

Figure 10. Convexity mechanism on the $\mathrm{Al}$ surface based on CAST.

\section{Conclusion}

In this study, we demonstrated the fabrication of a protrusion structure of Al-oxide containing SWCNTs on Al-plates through CAST processing. An alternating current flow was applied between the working (Al-plate) and counter electrodes in an alkaline aqueous solution in which SWCNTs were dispersed during the CAST process. The height and spacing of the protrusion structures ranged from $100-200 \mathrm{~nm}$ and $50-100 \mathrm{~nm}$, respectively. Furthermore, we investigated the formation mechanism of the protrusion structure through CAST processing and concluded that surface etching of the SWCNTs occurred when the working electrode was the cathode in the CAST process. Moreover, the $\mathrm{Al}^{3+}$ ions in the solution precipitated on the working electrode when it was the cathode. By repeating this process, protrusion structures composed of Al-oxides were formed on the Al-plates. In addition, SWCNTs were incorporated into the Al-oxide layer during precipitation. As a result, protrusion structures of Al-oxide containing SWCNTs were formed on the Al-plates.

\section{Acknowledgements}

This work was partly supported by the Ministry of Economy, Trade and Industry (METI) Monozukiri R \& D Support Grant Program for SMEs (Grant Number JPS005698). The results of this research are part of the results of the strategic basic technology advancement support project, "Research and development of inexpensive water-repellent treatment technology that reproduces the lotus leaf structure at the nano level by wet treatment." We would like to thank Nagoya Industrial Science Research Institute, Organization for Small \& Medium Enterprises and Regional Innovation, Japan, and KORBON Co. Ltd., Republic of Korea.

\section{Conflicts of Interest}

The authors declare no conflicts of interest regarding the publication of this paper.

\section{References}

[1] Ijima, S. (1991) Helical Microtubules of Graphitic Carbon. Nature (London), 354, 
56-58. https://doi.org/10.1038/354056a0

[2] Baughman, R.H., Zakhidov, A.A. and de Heer, W.A. (2002) Carbon Nanotubes the Route toward Applications. Science, 297, 787-792.

https://doi.org/10.1126/science.1060928

[3] Islam, M.S., Deng, Y., Tong, L., et al. (2016) Grafting Carbon Nanotubes Directly Onto Carbon Fibers for Superior Mechanical Stability: Towards Next Generation Aerospace Composites and Energy Storage Applications. Carbon, 96, 701-710. https://doi.org/10.1016/j.carbon.2015.10.002

[4] Wu, G.P., Wang, Y.Y., Li, D.H., et al. (2011) Direct Electrochemical Attachment of Carbon Nanotubes to Carbon Fiber Surfaces. Carbon, 49, 2152-2155.

https://doi.org/10.1016/j.carbon.2011.01.026

[5] Liu, Y.T., Wu, G.P. and Lu, C.X. (2016) Grafting of Carbon Nanotubes Onto Carbon Fiber Surfaces by Step-Wise Reduction of In-Situ Generated Diazonium Salts for Enhancing Carbon/Epoxy Interfaces. Materials Letters, 134, 75-79. https://doi.org/10.1016/j.matlet.2014.07.053

[6] Zhou, Z.W., Yan, Q.H., Liu, C.H., et al. (2017) An Arm-Like Electrothermal Actuator Based on Superaligned Carbon Nanotubes/Polymer Composites. New Carbon Materials, 32, 411-418. https://doi.org/10.1016/S1872-5805(17)60132-X

[7] Paul, S., Rajbongshi, B., Bora, B., et al. (2017) Organic Photovoltaic Cells Using MWCNTs. New Carbon Materials, 32, 27-34. https://doi.org/10.1016/S1872-5805(17)60104-5

[8] Mamalis, A.G., Vogtländer, L.O.G. and Markopoulos, A. (2004) Nanotechnology and Nanostructured Materials: Trends in Carbon Nanotubes. Precision Engineering, 28, 16-30. https://doi.org/10.1016/j.precisioneng.2002.11.002

[9] Baxendale, M. (2003) The Physics and Applications of Carbon Nanotubes. Journal of Materials Science: Materials in Electronics, 14, 657-659. https://doi.org/10.1023/A:1026158432563

[10] Thostenson, E.T., Ren, Z. and Chou, T.W. (2001) Advances in the Science and Technology of Carbon Nanotubes and Their Composites: A Review. Composites Science and Technology, 61, 1899-1912. https://doi.org/10.1016/S0266-3538(01)00094-X

[11] Lau, A.K. and Hui, D. (2002) The Revolutionary Creation of New Advanced Materials-Carbon Nanotube Composites. Composites Part B: Engineering, 33, 263-277. https://doi.org/10.1016/S1359-8368(02)00012-4

[12] Mauron, Ph., Emmenegger, Ch., Züttel, A., Nützenadel, Ch., Sudan, P. and Schlapbach, L. (2002) Synthesis of Oriented Nanotube Films by Chemical Vapor Deposition. Carbon, 40, 1339-1344. https://doi.org/10.1016/S0008-6223(01)00295-0

[13] Kim, P., Shi, L., Majumdar, A. and McEuen, P.L. (2001) Thermal Transport Measurements of Individual Multiwalled Nanotubes. Physical Review Letters, 87, Article ID: 215502. https://doi.org/10.1103/PhysRevLett.87.215502

[14] Luo, C., Zuo, X., Wang, L., Wang, E., Song, S., Wang, J., Fan, C. and Cao, Y. (2008) Flexible Carbon Nanotube-Polymer Composite Films with High Conductivity and Superhydrophobicity Made by Solution Process. Nano Letters, 8, 4454-4458. https://doi.org/10.1021/nl802411d

[15] Wei, C., Srivastava, D. and Cho, K. (2002) Thermal Expansion and Diffusion Coefficients of Carbon Nanotube-Polymer Composites. Nano Letters, 2, 647-650. https://doi.org/10.1021/nl025554+

[16] Liao, Q., Liu, Z., Liu, W., Deng, C. and Yang, N. (2015) Extremely High Thermal 
Conductivity of Aligned Carbon Nanotube-Polyethylene Composites. Scientific Reports, 5, Article No. 16543. https://doi.org/10.1038/srep16543

[17] Chu, K., Guo, H., Jia, C., Yin, F., Zhang, X., Liang, X. and Chen, H. (2010) Thermal Properties of Carbon Nanotube-Copper Composites for Thermal Management Applications. Nanoscale Research Letters, 5, Article No. 868.

https://doi.org/10.1007/s11671-010-9577-2

[18] Chen, P., Zhang, J., Shen, Q., Luo, G., Dai, Y., Wang, C., Li, M. and Zhang, L. (2017) Microstructure and Thermal Conductivity of Carbon Nanotube Reinforced $\mathrm{Cu}$ Composites. Journal of Nanoscience and Nanotechnology, 17, 2447-2452. https://doi.org/10.1166/jnn.2017.12637

[19] Chen, X., Bao, R., Yi, J., Fang, D., Tao, J. and Liu, Y. (2019) Enhancing Interfacial Bonding and Tensile Strength in CNT-Cu Composites by a Synergetic Method of Spraying Pyrolysis and Flake Powder Metallurgy. Materials (BaseI), 12, 670. https://doi.org/10.3390/ma12040670

[20] Nam, D.H., Kim, J.H., Cha, S.I., Jung, S.I., Lee, J.K., Park, H.M., Park, H.D. and Hong, H. (2014) Hardness and Wear Resistance of Carbon Nanotube Reinforced Aluminum-Copper Matrix Composites. Journal of Nanoscience and Nanotechnology, 14, 9134-9138. https://doi.org/10.1166/jnn.2014.10084

[21] Noguchi, T., Magario, A., Fukazawa, S., Shimizu, S., Beppu, J. and Seki, M. (2004) Carbon Nanotube/Aluminium Composites with Uniform Dispersion. Materials Transactions, 45, 602. https://doi.org/10.2320/matertrans.45.602

[22] Laha, T., Agarwal, A., McKechnie, T. and Seal, S. (2004) Synthesis and Characterization of Plasma Spray Formed Carbon Nanotube Reinforced Aluminum Composite. Materials Science and Engineering. A, 381, 249-258.

https://doi.org/10.1016/j.msea.2004.04.014

[23] Kuzumaki, T., Ujiie, O., Ichinose, H. and Ito, K. (2000) Mechanical Characteristics and Preparation of Carbon Nanotube Fiber-Reinforced Ti Composite. Advanced Engineering Materials, 2, 416-418. https://doi.org/10.1002/1527-2648(200007)2:7<416::AID-ADEM416>3.0.CO;2-Y

[24] Vincent, H., Vincent, C., Berthet, M.P., Bouix, J. and Gonzalez, G. (1996) Boron Carbide Formation from $\mathrm{BCl}_{3}-\mathrm{CH}_{4}-\mathrm{H}_{2}$ Mixtures on Carbon Substrates and in a Carbon Fibre Reinforced Al Composite. Carbon, 34, 1041-1055. https://doi.org/10.1016/0008-6223(96)00058-9

[25] Zhou, Y., Yang, W., Xia, Y. and Mallick, P.K. (2003) An Experimental Study on the Tensile Behavior of a Unidirectional Carbon Fiber Reinforced Aluminum Composite at Different Strain Rates. Materials Science and Engineering: A, 362, 112-117. https://doi.org/10.1016/S0921-5093(03)00214-4

[26] Kuzumaki, T., Miyazawa, K., Ichinose, H. and Ito, K. (1998) Processing of Carbon Nanotube Reinforced Aluminum Composite. Journal of Materials Research, 13, 2445-2449. https://doi.org/10.1557/JMR.1998.0340

[27] Xu, C.L., Wei, B.Q., Ma, R.Z., Liang, J., Ma, X.K. and Wu, D.H. (1999) Fabrication of Aluminum-Carbon Nanotube Composites and Their Electrical Properties. Carbon, 37, 855-858. https://doi.org/10.1016/S0008-6223(98)00285-1

[28] Zhong, R., Cong, H. and Hou, P. (2003) Fabrication of Nano-Al Based Composites Reinforced by Single-Walled Carbon Nanotubes. Carbon, 41, 848-851. https://doi.org/10.1016/S0008-6223(02)00427-X

[29] Iijima, S. and Ichihashi, T. (1993) Single-Shell Carbon Nanotubes of 1-nm Diameter. Nature, 363, 603-605. https://doi.org/10.1038/363603a0

[30] Saito, R., Dresselhaus, G. and Dresselhaus, M.S. (1998) Physical Properties of Car- 
bon Nanotubes. Imperial College Press, London. https://doi.org/10.1142/p080

[31] Geetha, M.B. and Rajendran, S. (2016) Synergistic Inhibition of Corrosion of Mild Steel in Sulphuric acid by New Ternary System. Der Pharma Chemica, 8, 194-201.

[32] Zhang, J.C., Tang, Y.J., Yi, Y., et al. (2016) Large-Scale Synthesis of Novel Vertically-Aligned Helical Carbon Nanotube Arrays. New Carbon Materials, 2, 213-223. https://doi.org/10.1016/S1872-5805(16)60032-X

[33] Wepasnick, K.A., Smith, B.A., Schrote, K.E., et al. (2011) Surface and Structural Characterization of Multi-Walled Carbon Nanotubes Following Different Oxidative Treatments. Carbon, 49, 24-36. https://doi.org/10.1016/j.carbon.2010.08.034

[34] Hirano, T., Nakade, K., Li, S., Kawai, K. and Arima, K. (2018) Chemical Etching of a Semiconductor Surface Assisted by Single Sheets of Reduced Graphene Oxide. Carbon, 127, 681-687. https://doi.org/10.1016/j.carbon.2017.11.053

[35] Jeong, S., Jung, S. and Lee, K. (2005) Preparation of Anodic Alumina Nanotemplate and Its Applications. Journal of Industrial and Engineering Chemistry, 16, 461-473.

[36] Masuda, H., Hasegwa, F. and Ono, S. (1997) Self-Ordering of Cell Arrangement of Anodic Porous Alumina Formed in Sulfuric Acid Solution. Journal of the Electrochemical Society, 144, L127-L130. https://doi.org/10.1149/1.1837634

[37] Martin, C.R. (1996) Membrane-Based Synthesis of Nanomaterials. Chemistry of Materials, 8, 1739-1746. https://doi.org/10.1021/cm960166s 